\title{
PERFIL DOS UROSTOMIZADOS CADASTRADOS EM UMA ASSOCIAÇÃO DE OSTOMIZADOS
}

Julliana Fernandes de Sena', Jéssika Wanessa Soares Costa', Lays Pinheiro de Medeiros' ${ }^{1}$ Cintia Galvão Queiroz', Samilly Márjore Dantas Liberato², Isabelle Katherinne Fernandes Costa ${ }^{3}$

${ }^{1}$ Discente de Enfermagem. Universidade Federal do Rio Grande do Norte. Natal-RN-Brasil.

${ }^{2}$ Enfermeira. Mestranda em Enfermagem. Universidade Federal do Rio Grande do Norte. Natal-RN-Brasil.

${ }^{3}$ Enfermeira. Doutora em Enfermagem. Docente da Universidade Federal do Rio Grande do Norte. Natal-RN-Brasil.

RESUMO: O objetivo deste estudo foi investigar o perfil dos urostomizados cadastrados na Associação dos Ostomizados do Rio Grande do Norte. Trata-se de um estudo descritivo, retrospectivo, desenvolvido entre outubro de 2013 a fevereiro de 2014, mediante informações colhidas nas fichas cadastrais de 53 pessoas com urostomia. A maior concentração de urostomizados cadastrados está localizada na zona litoral oriental do estado (60,4\%), com idade superior a 59 anos (73,6\%), pardos (50,9\%), do sexo masculino (54,7\%), casados (52,9\%), com ensino fundamental (35,9\%), com renda de um salário mínimo (56,6\%) e com diagnóstico de neoplasia de bexiga $(47,2 \%)$. Os resultados obtidos fornecem informações específicas sobre os urostomizados. Através da análise de informações sociais e demográficas e das condições de vida, é possível listar e priorizar os problemas desta população, colaborando, com a promoção da saúde, otimizando tempo e recursos financeiros.

DESCRITORES: Enfermagem; Estomia; Perfil de saúde.

\section{PROFILEOFUROSTOMIZEDPATIENTSREGISTERED IN AN ASSOCIATION OF OSTOMATES}

\begin{abstract}
The objective in this study was to investigate the profile of urostomized patients registered in the Association of Ostomates of Rio Grande do Norte. A descriptive and retrospective study was developed between October 2013 and February 2014, through information collected from the registration forms of 53 urostomized patients. The highest concentration of urostomized patients is located in the Eastern coastal region of the state $(60.4 \%)$, over 59 years of age $(73.6 \%)$, mulatto $(50.9 \%)$, male $(54.7 \%)$, married $(52.9 \%)$, who finished primary education $(35.9 \%)$, gain one minimum wage $(56.6 \%)$ and were diagnosed with bladder tumor $(47.2 \%)$. The results provide specific information on the urostomized patients. Through the analysis of social and demographic data and information about the living conditions, this population's problems can be listed in order of priority, contributing to health promotion and optimizing time and financial resources.
\end{abstract}

DESCRIPTORS: Nursing; Stoma; Health profile.

\section{PERFIL DE LOS UROSTOMIZADOS REGISTRADOS EN UNA ASOCIACIÓN DE OSTOMIZADOS}

RESUMEN: El objetivo de este estudio fue investigar el perfil de los urostomizados registrados en la Asociación de los Ostomizados de Rio Grande del Norte. Es un estudio descriptivo, retrospectivo, desarrollado entre octubre de 2013 y febrero de 2014, con informaciones obtenidas en las fichas de registro de 53 personas con urostomía. La mayor concentración de urostomizados registrados está ubicada en la zona litoral oriental del estado $(60,4 \%)$, con edad superior a 59 años (73,6\%), pardos (50,9\%), del sexo masculino (54,7\%), casados (52,9\%), enseñanza fundamental $(35,9 \%)$, renta de un salario mínimo $(56,6 \%)$ y con diagnóstico de neoplasia de vejiga (47,2\%). Los resultados obtenidos ofrecen informaciones específicas sobre los urostomizados. Por medio del análisis de informaciones sociales y demográficas y de las condiciones de vida, es posible enunciar y priorizar los problemas de esta población, colaborando con la promoción de la salud, otimizando tiempo y recursos financieros.

DESCRIPTORES: Enfermería; Estomía; Perfil de salud. 


\section{INTRODUÇÃO}

Mudanças do funcionamento do aparelho urinário ou intestinal podem resultar na criação de uma estomia ${ }^{(1)}$ devido à necessidade de desviar o trânsito normal das eliminações fisiológicas. Estoma é uma abertura criada artificialmente a partir do trato gastrointestinal (Gl) ou trato urinário, onde os resíduos do corpo iriam sair, numa superfície do corpo. Esta é uma abertura deliberada, feita a partir de um segmento do intestino, desviando o fluxo de fezes ou urina. Não há fornecimento de nervo sensorial ao estoma, as sensações como a dor não podem ser sentidas $^{(2)}$.

Urostomia é quando um segmento do intestino delgado é usado para formar um canal entre os ureteres e a parede abdominal, para permitir que a urina escorra por fora das vias naturais ${ }^{(3)}$.

Estima-se que em 2013 existam aproximadamente 700 mil ostomizados nos Estados Unidos da América ${ }^{(4)}$. No Brasil, existem cerca de 33.864 pessoas ostomizadas ${ }^{(5)}$, no Rio Grande do Norte $(\mathrm{RN})$, cerca 700 pessoas com ostomias $^{(6)}$.

Independentemente do tipo de ostomia, o paciente pode sentir-se diferente, visto que a mutilação no corpo e o uso do dispositivo coletor levam-no à necessidade de reconstrução da sua identidade. A perda de um órgão, perda da autoestima e autoconceito resultantes da imagem corporal, perda do seu status social, depressão, repulsa e sentimento de inutilidade são alguns dos anseios vivenciados pelo paciente com urostomia. Acrescentam-se, alterações na vida sexual, diminuição da libido e preocupações com a eliminação de odores, fezes ou urina durante a relação sexual. Neste momento o apoio dos familiares e amigos, assim como dos profissionais de saúde é fundamental para os sujeitos urostomizados ${ }^{(7)}$.

No Rio Grande do Norte, os ostomizados cadastrados recebem o apoio da Associação dos Ostomizados do Rio Grande do Norte (AORN), fundada em 1991, atualmente com mais de 800 associados ativos em atendimento, ajudando a promover a reintegração dos ostomizados na vida cotidiana ${ }^{(8)}$. Apesar da existência de centros de referências no Nordeste, o acervo de material para pesquisa sobre o assunto ainda é escasso.
Os estudos publicados que envolvam a temática, no RN, são exíguos, e o perfil destes pacientes é limitado, o que nos fez refletir e levantar a seguinte questão de pesquisa: Qual o perfil dos urostomizados cadastrados na AORN?

Dentro deste contexto, o objetivo deste estudo foi investigar o perfil dos urostomizados ativos cadastrados na AORN. Este estudo oportunizará a equipe de saúde, em especial o enfermeiro, a partir do perfil identificado, aperfeiçoar o planejamento das ações assistenciais de modo integral por meio do conhecimento que o profissional interpreta e com o qual estabelece novos direcionamentos para sua prática, além de promover o aprimoramento da relação profissional-paciente.

\section{MÉTODO}

Trata-se de um estudo descritivo, retrospectivo, desenvolvido entre outubro de 2013 e fevereiro de 2014 na AORN, que abrange todos os municípios desse estado. A população do estudo compreende todos os pacientes urostomizados ativos cadastrados no banco de dados da associação.

Os dados foram obtidos por consulta aos documentos impressos denominados de ficha cadastral, individual para cada paciente ostomizado, preenchida no momento em que o mesmo compareceu à referida associação com o objetivo de cadastramento, possibilitando a obtenção das bolsas de ostomia.

As variáveis estudadas foram: sexo, cor, procedência, estado civil, renda, profissão/ ocupação, escolaridade, diagnóstico, motivo da realização da ostomia, tipo de estoma e duração da ostomia. Para o banco de dados e análise utilizou-se tratamento estatístico através do programa de informática Microsoft Office Excel $2010^{\circledR}$ com estatística descritiva.

Conforme prevê a Resolução 466/12 do Conselho Nacional da Saúde, a consulta aos impressos foi realizada após autorização da diretoria da referida associação e após aprovação do Comitê de Ética em Pesquisa/UFRN (CAAE 19866413.3.0000.5537). Todos os preceitos éticos de pesquisa em seres humanos foram atendidos. 


\section{RESULTADOS}

Dos 697 pacientes ativos cadastrados na AORN, 53 (7,6\%) possuíam urostomias. Destes $\mathrm{n}=39(73,6 \%)$ possuíam mais de 59 anos, eram do sexo masculino $n=29(54,7 \%)$, com ascendência de pardos $n=27(50,9 \%)$ e prevalência de casados $\mathrm{n}=28(52,9 \%)$, conforme Tabela 1 . Destaca-se o elevado percentual de fichas cadastrais que não possuíam informações completas, tidas como dados ignorados neste estudo.

Em relação à escolaridade, destacase a quantidade de pacientes com ensino fundamental, predominando urostomizados acima de 59 anos $n=19$ (35,9\%). Dados referentes à profissão demonstraram que $n=14(26,4 \%)$ era, de aposentados, pensionistas ou beneficiários. Quanto à renda, $\mathrm{n}=30(56,6 \%)$ tem ganho de até um salário mínimo (SM), como exposto na Tabela 2.

No que concerne à causa da realização da urostomia, a Tabela 3 demonstra que $n=25(47,2 \%)$ havia sido diagnosticada com neoplasia de bexiga. No entanto, um paciente apresentava urostomia com ileostomia com o diagnóstico relacionado a carcinoma urotelial. Seis pacientes apresentavam urostomia com colostomia com diagnóstico de neoplasia de reto. Quanto à duração da ostomia, constatou-se que $n=50(94,3 \%)$ dos urostomizados possuíam ostomias definitivas, desses $n=38$ $(71,7 \%)$ na faixa etária acima de 59 anos.

No que se refere à região com maior concentração de associados com urostomia, verificou-se que a maioria deles estava localizada na zona litoral oriental $n=32(60,4 \%)$, seguida da zona mossoroense $n=9(17 \%)$, conforme demonstrado na Figura 1.

Tabela 1 - Características sociodemográficas de pacientes com urostomia segundo a faixa etária. Natal-RNBrasil, 2014

\begin{tabular}{lcccccc}
\hline Características & \multicolumn{2}{c}{ Até $\mathbf{5 9}$ anos } & \multicolumn{2}{c}{$\boldsymbol{>} \mathbf{5 9}$ anos } & \multicolumn{2}{c}{ Total } \\
\cline { 2 - 7 } Sociodemográficas & $\mathbf{n}$ & $\mathbf{\%}$ & $\mathbf{n}$ & $\mathbf{0}$ & $\mathbf{n}$ & $\mathbf{\%}$ \\
\hline Sexo & & & & & & \\
\hline Masculino & 8 & 15,1 & 21 & 39,6 & 29 & 54,7 \\
\hline Feminino & 6 & 11,3 & 18 & 34 & 24 & 45,3 \\
\hline Cor & & & & & \\
\hline Branco & 7 & 13,2 & 13 & 24,5 & 20 & 37,7 \\
\hline Pardo & 6 & 11,3 & 21 & 39,6 & 27 & 50,9 \\
\hline Preto & 1 & 1,9 & 4 & 7,6 & 5 & 9,5 \\
\hline Ignorado & 0 & 0 & 1 & 1,9 & 1 & 1,9 \\
\hline Estado civil & & & & & & \\
\hline Casado & 7 & 13,2 & 21 & 39,6 & 28 & 52,9 \\
\hline Solteiro & 6 & 11,3 & 5 & 9,5 & 11 & 20,7 \\
\hline Viúvo & 1 & 1,9 & 8 & 15,1 & 9 & 17 \\
\hline Separado & 0 & 0 & 4 & 7,5 & 4 & 7,5 \\
\hline Ignorado & 0 & 0 & 1 & 1,9 & 1 & 1,9 \\
\hline Total & 14 & 26,4 & 39 & 73,6 & 53 & 100 \\
\hline & & & & &
\end{tabular}


Tabela 2 - Características sociodemográficas de pacientes com urostomia segundo a faixa etária. Natal-RNBrasil, 2014

\begin{tabular}{|c|c|c|c|c|c|c|}
\hline \multirow{2}{*}{$\begin{array}{l}\text { Características } \\
\text { Sociodemográficas }\end{array}$} & \multicolumn{2}{|c|}{ Até 59 anos } & \multicolumn{2}{|c|}{$>59$ anos } & \multicolumn{2}{|c|}{ Total } \\
\hline & $\mathbf{n}$ & $\%$ & $\mathbf{n}$ & $\%$ & $\mathbf{n}$ & $\%$ \\
\hline \multicolumn{7}{|l|}{ Escolaridade } \\
\hline Analfabeto & 4 & 7,5 & 13 & 24,5 & 17 & 32 \\
\hline Ensino fundamental & 9 & 17 & 19 & 35,9 & 28 & 52,9 \\
\hline $\begin{array}{l}\text { Ensino médio } \\
\text { incompleto }\end{array}$ & 1 & 1,9 & 1 & 1,9 & 2 & 3,8 \\
\hline Ensino superior & 0 & 0 & 4 & 7,5 & 4 & 7,5 \\
\hline Ignorado & 0 & 0 & 2 & 3,8 & 2 & 3,8 \\
\hline \multicolumn{7}{|l|}{ Profissão } \\
\hline $\begin{array}{l}\text { Aposentado/ } \\
\text { pensionista/beneficiário }\end{array}$ & 3 & 5,7 & 11 & 20,7 & 14 & 26,4 \\
\hline Do lar & 3 & 5,7 & 4 & 7,5 & 7 & 13,2 \\
\hline Outros & 3 & 5,7 & 2 & 3,8 & 5 & 9,5 \\
\hline Agricultor/pescador & 0 & 0 & 3 & 5,7 & 3 & 5,7 \\
\hline Militar/marítimo & 1 & 1,9 & 1 & 1,9 & 2 & 3,8 \\
\hline Motorista & 0 & 0 & 1 & 1,9 & 1 & 1,9 \\
\hline Funcionário público & 0 & 0 & 1 & 1,9 & 1 & 1,9 \\
\hline Ignorado & 4 & 7,5 & 16 & 30,1 & 20 & 37,6 \\
\hline \multicolumn{7}{|l|}{ Renda familiar } \\
\hline $0 \mathrm{sm}$ & 1 & 1,9 & 2 & 3,8 & 3 & 5,7 \\
\hline Até $1 \mathrm{sm}$ & 12 & 22,6 & 18 & 33,9 & 30 & 56,6 \\
\hline Até $2 \mathrm{sm}$ & 0 & 0 & 9 & 17 & 9 & 17 \\
\hline Até $3 \mathrm{sm}$ & 1 & 1,9 & 3 & 5,7 & 4 & 7,5 \\
\hline$\geq 5 \mathrm{Sm}$ & 0 & 0 & 4 & 7,5 & 4 & 7,5 \\
\hline Ignorado & 0 & 0 & 3 & 5,7 & 3 & 5,7 \\
\hline Total & 14 & 26,4 & 39 & 73,6 & 53 & 100 \\
\hline
\end{tabular}

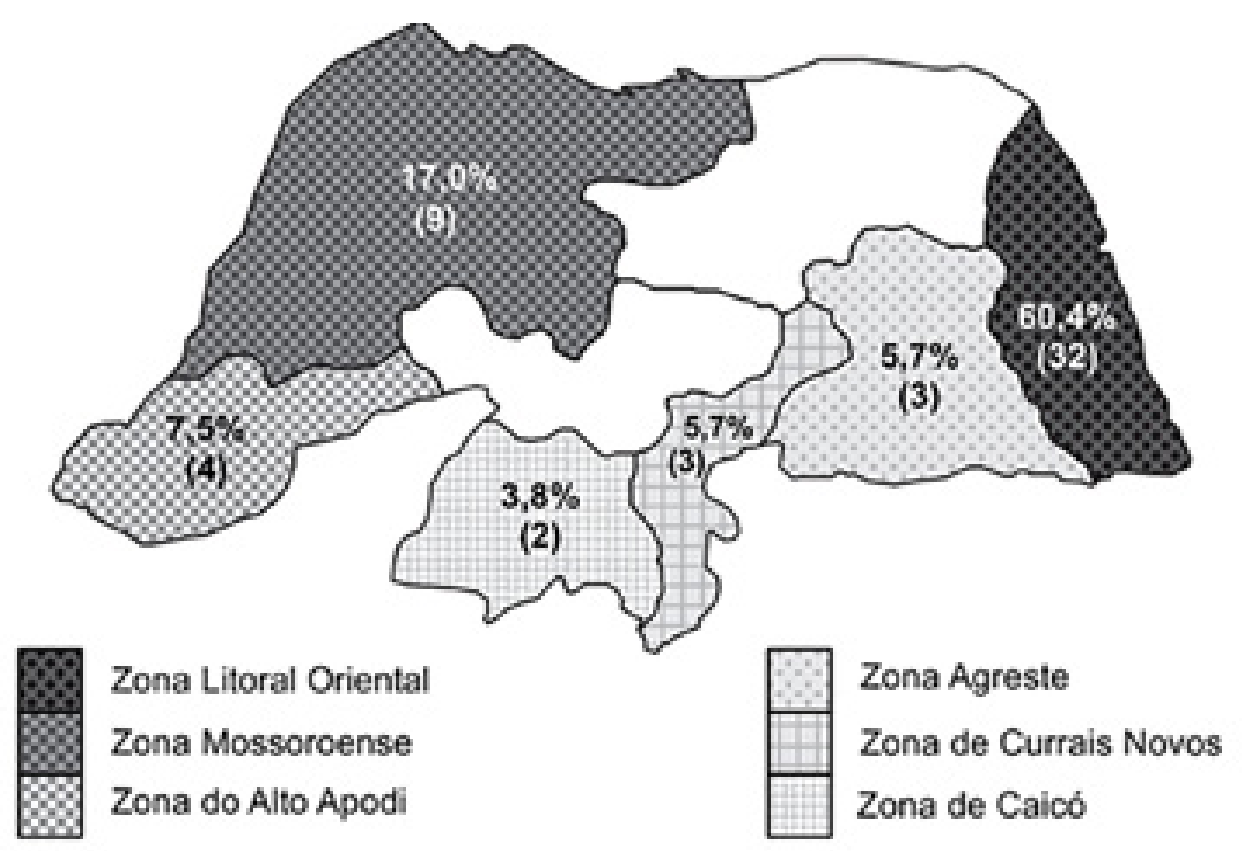

Figura 1 - Mapa geográfico do Rio Grande do Norte referente à distribuição dos urostomizados cadastrados na AORN. Natal-RN-Brasil, 2014 
Tabela 3 - Distribuição dos urostomizados quanto ao diagnóstico e duração da ostomia. Natal-RN-Brasil, 2014

\begin{tabular}{|c|c|c|c|c|c|c|}
\hline \multirow{2}{*}{ Variável } & \multicolumn{2}{|c|}{ Até 59 anos } & \multicolumn{2}{|c|}{$>59$ anos } & \multicolumn{2}{|c|}{ Total } \\
\hline & $\mathbf{n}$ & $\%$ & $\mathbf{n}$ & $\%$ & $\mathbf{n}$ & $\%$ \\
\hline \multicolumn{7}{|l|}{ Diagnóstico } \\
\hline Neoplasia de reto & 1 & 1,9 & 2 & 3,8 & 3 & 5,7 \\
\hline $\begin{array}{l}\text { Neoplasia de Colo } \\
\text { Uterino }\end{array}$ & 1 & 1,9 & 2 & 3,8 & 3 & 5,7 \\
\hline $\begin{array}{l}\text { Neoplasia de Cólon } \\
\text { (intestinal) }\end{array}$ & 1 & 1,9 & 0 & 0 & 1 & 1,9 \\
\hline Lesão de Bexiga & 1 & 1,9 & 3 & 5,7 & 4 & 7,5 \\
\hline Fístula Vesicovaginal & 1 & 1,9 & 1 & 1,9 & 2 & 3,8 \\
\hline Neoplasia de Bexiga & 4 & 7,5 & 21 & 39,6 & 25 & 47,2 \\
\hline $\begin{array}{l}\text { Insuficiência Renal } \\
\text { Crônica }\end{array}$ & 1 & 1,9 & 0 & 0 & 1 & 1,9 \\
\hline Carcinoma Urotelial & 1 & 1,9 & 3 & 5,7 & 4 & 7,5 \\
\hline Hidronefrose & 1 & 1,9 & 0 & 0 & 1 & 1,9 \\
\hline $\begin{array}{l}\text { Ferimento por Arma de } \\
\text { Fogo (FAF) }\end{array}$ & 2 & 3,8 & 0 & 0 & 2 & 3,8 \\
\hline $\begin{array}{l}\text { Neoplasia de Colo } \\
\text { Uterino/Fístula }\end{array}$ & 0 & 0 & 1 & 1,9 & 1 & 1,9 \\
\hline Vesicovaginal & 0 & 0 & 1 & 1,9 & 1 & 1,9 \\
\hline Cisto Prostático & 0 & 0 & 1 & 1,9 & 1 & 1,9 \\
\hline Fístula Retovaginal & 0 & 0 & 1 & 1,9 & 1 & 1,9 \\
\hline Carcinoma & 0 & 0 & 1 & 1,9 & 1 & 1,9 \\
\hline Carcinoma Ureteral & 0 & 0 & 1 & 1,9 & 1 & 1,9 \\
\hline $\begin{array}{l}\text { Lesão Extensiva } \\
\text { Pélvica }\end{array}$ & 0 & 0 & 1 & 1,9 & 1 & 1,9 \\
\hline Tumor Renal & 0 & 0 & 1 & 1,9 & 1 & 1,9 \\
\hline \multicolumn{7}{|l|}{ Duração } \\
\hline Definitiva & 12 & 22,6 & 38 & 71,7 & 50 & 94,3 \\
\hline Temporária & 2 & 3,8 & 0 & 0 & 2 & 3,8 \\
\hline Ignorado & 0 & 0 & 1 & 1,9 & 1 & 1,9 \\
\hline Total & 14 & 26,4 & 39 & 73,6 & 53 & 100 \\
\hline
\end{tabular}

\section{DISCUSSÃO}

Os dados demográficos apresentados neste estudo corroboram com o processo de transição demográfica que está ocorrendo no Brasil, caracterizando o envelhecimento populacional como um fato relevante no contexto de assistência à saúde, visto que demonstra também uma transição do modelo assistencial, caracterizado pela crescente necessidade da assistência hospitalar e seus respectivos níveis de complexidade. Diversos são os fatores que afetam a qualidade de vida (QV) do idoso, sendo alguns deles relacionados com as características sociodemográficas, como faixa etária, classe econômica, escolaridade, estado civil e situação ocupacional, e outros associados às condições de saúde, representados pela classificação do Índice de Massa Corpórea (IMC), problemas de saúde e uso de medicamentos ${ }^{(9-10)}$.

Quanto ao estado civil, os casados compõem a maioria deste estudo. Nesse sentido, como o cônjuge se insere como componente fundamental no processo adaptativo da pessoa com ostomia, uma vez que ocorrem inúmeras modificações físicas e psicológicas após a realização do procedimento. A partir da convivência, os familiares podem fornecer informações importantes, como hábitos e preferências, que auxiliem a formulação e execução de um plano terapêutico, e na interpretação de como este deve ser realizado, não só durante a fase adaptativa, 
mas em qualquer etapa do processo de saúde/ doença ${ }^{(11-12)}$.

Sendo assim, frente aos relatos dos ostomizados que descrevem sentir vergonha, medo, insegurança e constrangimento, a família se constitui como um suporte de apoio para promover a melhora da autoestima e reinserção da pessoa com ostomia na sociedade ${ }^{(13)}$.

A baixa escolaridade dos urostomizados apresentada neste estudo pode representar um obstáculo para o entendimento sobre sua condição de saúde e realização das ações de autocuidado, uma vez que a maioria dos indivíduos entrevistados enquadra-se na categoria de ensino fundamental. $\mathrm{O}$ acesso às informações e aos serviços de saúde, assim como a outros recursos da comunidade estão diretamente relacionados ao nível socioeconômico e cultural. Há, portanto, influência do conhecimento sobre a necessidade de realização de exames de rotina para detecção precoce do câncer, por exemplo, na busca por atendimento e oportunidades de acesso aos recursos médico/hospitalares ${ }^{(14)}$.

A maioria dos pacientes urostomizados era composta por aposentados, seguida de agricultores e do lar. Com a publicação da Lei Federal 5.296, que classifica os ostomizados como deficientes físicos, ocorre a inserção dessa clientela na aposentadoria, em conjunto com as demais leis que regulamentam os direitos das pessoas com deficiência no Brasil, nas esferas federal, estadual e municipal(15).

Dentre esses direitos, destacam-se o amparo assistencial ao idoso e ao deficiente, representado pela Lei Orgânica da Assistência Social (LOAS), que fornece o benefício de um salário mínimo mensalmente, a partir da comprovação de que não possui meios de prover à sua própria manutenção ou tê-la provida por sua família; isenção de imposto de renda na aposentadoria por invalidez; saque do Programa de Integração Social (PIS) e do Fundo de Garantia do Tempo de Serviço (FGTS); isenção de Imposto sobre Operações relativas à Circulação de Mercadorias e sobre Prestações de Serviços de Transporte Interestadual, Intermunicipal e de Comunicação (ICMS) e Imposto Sobre Produtos Industrializados (IPI), na compra de carro adaptado; isenção de Imposto sobre Propriedade de Veículos Automotores (IPVA) para veículos adaptados; auxílio-doença; BPC-LOAS (Benefício de
Prestação Continuada da Assistência Social); passe livre municipal, intermunicipal e interestadual ${ }^{(15)}$.

No que se refere as questões financeiras, vale ressaltar que a urostomia representa uma fonte adicional de gastos. As formas que ela afeta a saúde podem ser entendidas pelo uso da renda na aquisição de bens e serviços de saúde, condições de moradia e educação ${ }^{(16)}$.

Sobre os diagnósticos associados à realização da urostomia, a neoplasia de bexiga destaca-se em relação aos demais. O surgimento de uma neoplasia pode ocasionar sofrimento e dor ao indivíduo, gerando instabilidade emocional durante a vivência com a nova condição e seu tratamento ${ }^{(17)}$. Vários são os fatores de risco que podem estar associados à incidência de desta neoplasia, destaca-se o tabagismo como o maior fator de risco para seu desenvolvimento, com um risco duas a quatro vezes maior que os não fumantes, com duplo risco se esta neoplasia estiver relacionada à concomitância entre tabaco e sobrepeso ${ }^{(18-19)}$.

Os riscos ocupacionais também estão representados na literatura como fator de risco, em função da exposição a uma variedade de produtos químicos, que incluem hidrocarbonetos aromáticos policíclicos, formaldeídos e solventes. Há também a carga genética, infecções do trato urinário e a idade constituem-se como fatores adicionais que favorecem essa neoplasia ${ }^{(20-22)}$.

As ostomias podem ser classificadas em temporárias ou definitivas, as últimas representaram a maior parte dentre os tipos demonstrados neste estudo, corroborando com estudos internacionais que também obtiveram esse resultado. O processo adaptativo do ostomizado exige tempo, e desenvolvimento de habilidades específicas para a realização do autocuidado e superação de obstáculos causados por essa nova condição física, psicológica e fisiológica. Nesse sentido, pessoas com ostomias definitivas se diferenciam das com as temporárias pela necessidade permanente desse ajustamento, em que o tempo influencia na maneira como essas pessoas enfrentam essa situação ${ }^{(23-24)}$.

Quanto à região de procedência dos associados com urostomia, identificou-se alta densidade populacional desse grupo na capital do estado, assim como em suas imediações, levando-nos a associar esse dado à localização da AORN, favorecendo o acesso aos recursos 
físicos e humanos, e à população relativa da região litoral oriental $(48,5 \%$ da população do $\mathrm{RN})^{(25)}$. Do mesmo modo, esse dado provoca uma reflexão sobre a dificuldade dos demais com urostomia, advindas demais regiões do estado, para o acesso à associação do Estado do RN e, consequentemente, às orientações de cuidado e manejo com as bolsas de ostomia, assim como para o fornecimento dessas.

\section{CONCLUSÃO}

Prevaleceram pessoas do sexo masculino, pardas, com união estável e idade superior a 59 anos. Tendo como renda familiar um salário mínimo, estando possivelmente associada à questão de serem maiores de 59 anos, e estarem inclusos no grupo dos aposentados, pensionistas ou beneficiários. Quanto à escolaridade, predominou dentre os urostomizados o ensino fundamental. Em relação à distribuição demográfica, os indivíduos deste estudo apresentam alta concentração na zona litoral oriental do RN.

Importante ressaltar que, conforme o exposto nos resultados, uma das limitações da pesquisa centrou-se na falta de informações na ficha cadastral de dados dos pacientes pesquisados referentes a algumas variáveis do estudo, como: cor, idade, renda, escolaridade, profissão, diagnóstico e tipo de estoma, que em alguns casos não estavam preenchidos, tidos neste estudo como dados ignorados.

Tendo em vista a temática urostomia, acreditase que esta pesquisa possa trazer subsídios para a reflexão acerca deste tema e suscitar o desenvolvimento de pesquisas futuras, uma vez que a literatura sobre essa área ainda é escassa. Contudo, o perfil dos urostomizados auxiliará a prática clínica, com a identificação das especificidades da população de ostomizados, colaborando com a promoção da saúde, otimizando tempo e recursos financeiros.

\section{REFERÊNCIAS}

1. Barros EJL, Santos SSC, Gomes GC, Erdmann AL. Gerontotecnologia educativa voltada ao idoso estomizado à luz da complexidade. Rev. gaúch. enferm. [Internet] 2012; 33(2): 95-101 [acesso em 10 set 2014]. Disponível: http://www.scielo.br/scielo.php?script=sci_ arttext\&pid=S1983-14472012000200014
2. Bartle C, Darbyshire M, Gaynor P, Hassan C, Whitfield J, Gardiner A. Addressing common stoma complications. Nursing \& resident. care. [Internet] 2013; 15(3) [acesso em 10 set 2014]. Disponível: http:// dx.doi.org/10.12968/nrec.2013.15.3.128

3. Slater R. Choosing one- and two-piece appliances. Nursing \& resident. care. [Internet] 2012; 14(8) [acesso em 10 set 2014]. Disponível: http://dx.doi. org/10.12968/nrec.2012.14.8.410.

4. United Ostomy Associations of America (UOAA) [Internet] [acesso em 20 abr 2014]. Disponível: http:// www.ostomy.org

5. Associação Brasileira de Ostomizados (ABRASO). Quantitativo aproximado de Pessoas Ostomizadas no Brasil [Internet] 2007 [acesso em 17 abr 2014]. Disponível: http://www.abraso.org.br/estatistica_ ostomizados.htm

6. Sena JF, Queiroz CG, Freitas LS, Andrade RS, Costa IKF. Perfil dos usuários cadastrados na associação de ostomizados do Rio Grande do Norte. I Congresso Nacional Ciências da Saúde- CONACIS; Mar. de 2014, Cajazeiras, PB, Brasil. Editora Realize; [Internet] 2014 [acesso em 08 set 2014]. Disponível: http:// www.editorarealize.com.br/revistas/conacis/resumo. php?idtrabalho $=260$

7. Bicalho MB, Lopes MHBM. Impacto da incontinência urinária na vida de esposas de homens com incontinência: revisão integrativa. Rev. esc. enferm. USP. [Internet] 2012; 46 (4) [acesso em 10 set 2014]. Disponível: http://dx.doi.org/10.1590/S008062342012000400032

8. Associação dos Ostomizados do Rio Grande do Norte (AORN). [Internet] 2012 [acesso em 03 abr 2014]. Disponível: http://aornnatal.com/

9. Vagetti GC, Filho VCB, Moreira NB, Oliveira V, Mazzardo O, Campos W. Condições de saúde e variáveis sociodemográficas associadas à qualidade de vida em idosas de um programa de atividade física de Curitiba, Paraná, Sul do Brasil. Cad. saúde pública [Internet] 2013; 29(5) [acesso em 10 set 2014]. Disponível: http://dx.doi.org/10.1590/S0102311X2013000900013

10. Mendes ACG, Sá DA, Miranda GMD, Lyra TM, Tavares RAW. Assistência pública de saúde no contexto da transição demográfica brasileira: exigências atuais e futuras. Cad. saúde pública [Internet] 2012; 28(5) [acesso em 10 set 2014]. Disponível: http://dx.doi. org/10.1590/S0102-311X2012000500014

11. Dornelles SSD, Silva DMGV, Mattosinho MMS, Kuhen AEK. O Cuidado à pessoa com Diabetes Mellitus e sua família. Cogitare enferm. [Internet] 2013 Jul/Set; 18(3) [acesso em 11 set 2014]. Disponível: http://ojs.c3sl.ufpr. br/ojs2/index.php/cogitare/article/view/33562/21059 
12. Cetolin S, Beltrame V, Presta A. Dinâmica sóciofamiliar com pacientes portadores de ostomia intestinal definitiva. ABCD arq. bras. cir. dig. [Internet] 2013; 26(3): 170-2 [acesso em 15 abr 2014]. Disponível: http://www.scielo.br/pdf/abcd/v26n3/03.pdf

13. Lenza NFB, Sonobe HM, Zago MMF, Buetto LS. Características socioculturais e clínicas de estomizados intestinais e de familiares em um programa de ostomizados. REE [Internet] 2013; 15(3) [acesso em 10 abr 2014]. Disponível: http://dx.doi.org/10.5216/ ree.v15i3.17594

14. Presidência da República (BR). Decreto n. 5296, de 02 de dezembro de 2004 Regulamenta as Leis n. 10.048, de 8 de novembro de 2000, que dá prioridade de atendimento às pessoas que especifica; e 10.098, de 19 de dezembro de 2000, que estabelece normas gerais e critérios básicos para a promoção da acessibilidade. [Internet] 2004 [acesso em 06 mai 2014]. Disponível: http://www.planalto.gov.br/ccivil_03/_ato20042006/2004/decreto/d5296.htm

15. Santos AMA, Jacinto PA, Tejada CAO. Causalidade entre renda e saúde: uma análise através da abordagem de dados em painel com os estados do Brasil. Estud. Econ. [Internet] 2012; 42(2) [acesso em 11 set 2014]. Disponível: http://dx.doi.org/10.1590/S010141612012000200001

16. Salimena AMO, Teixeira SR, Amorim TV, Paiva ACPC, Melo MCSC. O vivido dos enfermeiros no cuidado ao paciente oncológico. Cogitare enferm. [Internet] 2013 Jan/Mar; 18(1) [acesso em 11 set 2014]. Disponível: http://ojs.c3sl.ufpr.br/ojs2/index.php/cogitare/article/ view/31320/20027

17. Colli Neto JA, Zen Júnior JH, Negro AD, Andreollo NA, Araújo MR, Tincani AJ. Modelo experimental de tabagismo para indução de neoplasia da bexiga urinária. Rev. Col. Bras. Cir. [Internet] 2014; 41(1) [acesso em 11 de set 2014]. Disponível: http://dx.doi. org/10.1590/S0100-69912014000100011

18. Wyszynski S, Tanyos SA, Rees JR, Marsit CJ, Kelsey $K T$, Schned AR, et al. Body mass and smoking are modifiable risk factors for recurrent bladder cancer. [Internet] 2014; 120(3): [acesso em 10 mai 2014]. Disponível: http://onlinelibrary.wiley.com/doi/10.1002/ cncr.28394/abstract

19. Goossens-Laan CA, Kil PJ, Ruud Bosch JL, De Vries J. Pre-diagnosis quality of life $(\mathrm{QoL})$ in patients with hematuria: comparison of bladder cancer with other causes. Qual. Life Res. 2013; 22(2): 309-15.

20. Ministério da Saúde (BR). Instituto Nacional de Câncer (INCA). Estimativa 2014: Estimativa de Câncer no Brasil. [Internet] 2014 [acesso em 28 mai 2014]. Disponível: http://www.inca.gov.br/estimativa/2014/
Britain. Br. j. cancer. [Internet] 2012; 107(1) [acesso em 03 jun 2014]. Disponível: http://www.ncbi.nlm. nih.gov/pmc/articles/PMC3384013/?tool=pubmed

22. Martins AK, Oliveira Filho AA. Perfil epidemiológico dos casos de infecções do trato urinário (ITU) registrados no hospital regional de cajazeiras Paraíba. BioFar [Internet] 2012; 7(2) [acesso em 10 mai 2014] Disponível: http://sites.uepb.edu.br/biofar/ download/v7n2-2012/perfilepidemiologico.pdf

23. Nascimento CMS, Trindade GLB, Luz MHBA, Santiago RF. Vivência do paciente estomizado: uma contribuição para a assistência de enfermagem. Texto \& contexto enferm. [Internet] 2011; 20(3) [acesso em 03 mai 2014]. Disponível: http://dx.doi.org/10.1590/ S0104-07072011000300018

24. Sun V, Grant M, Mc Mullen CK, Altschuler A, Mohler MJ, Hornbrook MC, et al. Surviving colorectal cancer: long-term, persistent ostomy-specific concerns and adaptations. J Wound Ostomy Continence Nurs. 2013; 40(1): 61-72.

25. Instituto Brasileiro de Geografia e Estatística (IBGE). [Internet] Cidades [acesso em 05 mai 2014]. Disponível: http://www.censo2010.ibge.gov.br/sinopse/index. php?dados $=21 \& u f=24$

21. Brown T, Slack R, Rushton L. Occupational cancer in 